\title{
PTEN Inhibits Cell Proliferation, Promotes Cell Apoptosis, and Induces Cell Cycle Arrest via Downregulating the PI3K/AKT/hTERT Pathway in Lung Adenocarcinoma A549 Cells
}

\author{
Xiao-Xiao Lu, ${ }^{1}$ Lan-Yu Cao, ${ }^{1}$ Xi Chen, ${ }^{2}$ Jian Xiao, ${ }^{1}$ Yong Zou, ${ }^{1}$ and Qiong Chen ${ }^{1}$ \\ ${ }^{1}$ Department of Geriatrics, Xiangya Hospital of Central South University, Changsha, Hunan 410008, China \\ ${ }^{2}$ Department of Respiratory Medicine, Xiangya Hospital of Central South University, Changsha, Hunan 410008, China \\ Correspondence should be addressed to Qiong Chen; qiongch@163.com
}

Received 27 June 2016; Revised 12 September 2016; Accepted 20 September 2016

Academic Editor: Manoor Prakash Hande

Copyright (c) 2016 Xiao-Xiao Lu et al. This is an open access article distributed under the Creative Commons Attribution License, which permits unrestricted use, distribution, and reproduction in any medium, provided the original work is properly cited.

PTEN plays an essential role in tumorigenesis and both its mutation and inactivation can influence proliferation, apoptosis, and cell cycle progression in tumor cells. However, the precise role of PTEN in lung cancer cells has not been well studied. To address this, we have generated lung adenocarcinoma A549 cells overexpressing wild-type or mutant PTEN as well as A549 cells expressing a siRNA directed toward endogenous PTEN. Overexpression of wild-type PTEN profoundly inhibited cell proliferation, promoted cell apoptosis, caused cell cycle arrest at G1, downregulated p-AKT, and decreased expression of the telomerase protein $h T E R T$. In contrast, in cells expressing a PTEN directed siRNA, the opposite effects on cell proliferation, apoptosis, cell cycle arrest, pAKT levels, and $h T E R T$ protein expression were observed. A549 cells transfected with a PTEN mutant lacking phosphatase activity (PTEN-C124A) or an empty vector (null) did not show any effect. Furthermore, using the PI3K/AKT pathway blocker LY294002, we confirmed that the PI3K/AKT pathway was involved in mediating these effects of PTEN. Taken together, we have demonstrated that PTEN downregulates the PI3K/AKT/hTERT pathway, thereby suppressing the growth of lung adenocarcinoma cells. Our study may provide evidence for a promising therapeutic target for the treatment of lung adenocarcinoma.

\section{Introduction}

Both oncogenes and tumor suppressors are potential targets in the field of tumor therapy. In organisms, the biological functions of oncogenes and antioncogenes mutually antagonize each other to regulate cell proliferation, differentiation, apoptosis, cell cycle, and angiogenesis. It has been found that dozens of genes are closely correlated with lung cancer, among which the oncogene PTEN (phosphatase and tensin homolog) and the tumor suppressor hTERT (human telomerase reverse transcriptase) have been extensively studied in the past few years [1-4].

The tumor suppressor gene PTEN encodes dual-specificity phosphatase that was first discovered in 1997 [5]. Inactivation of PTEN is a key event in tumorigenesis and tumor development, and in fact it has the highest frequency of mutation in cancer after the P53 gene [6]. Currently, the tumor suppressing mechanism of the PTEN gene likely involves several candidate pathways, including the FAK pathway [7], the MAPK pathway $[8,9]$, and the PI3K/AKT pathway $[10,11]$. Currently, the PI3K/AKT pathway is regarded as the key pathway by which PTEN exerts its antioncogenic effects. PTEN encodes a protein with lipid phosphatase activity, which can dephosphorylate PIP3 (phosphatidylinositol $(3,4,5)$-trisphosphate) to form PIP2 (phosphatidylinositol $(4,5)$-bisphosphate), thereby preventing growth factor signal transduction pathways regulated by PI3K/AKT. As a result, PTEN activity in tumor cells results in cell cycle arrest at the G1 phase and induction of apoptosis [10-12]. Moreover, the PI3K/AKT pathway plays an important central role in tumor progression, and it is closely associated with other pathways which control a wide variety of tumor related biological processes. Studies have found that both the FAK pathway and the MAPK pathway exert effects through the PI3K/AKT pathway and affect the activity of AKT [7-9]. In a study of ovarian cancer, it was found that the FAK pathway mediated 
the activation of multiple downstream substrates of AKT such as NF- $\kappa \mathrm{B}$, promoted cell proliferation, and improved resistance to chemotherapeutic drugs [13].

Numerous studies have shown that telomerase activation is another extremely important step in the pathogenesis of lung cancer, and this depends on the activity of the hTERT gene $[14,15]$. Despite its importance, the mechanisms of hTERT gene regulation have not been completely identified. However, it has been shown that there is a negative correlation between $h T E R T$ expression and PTEN expression in gastric cancer, liver cancer, and endometrial cancer [16].

PTEN has been found to be able to inhibit the activity of telomerase. The activity of telomerase declined significantly when wild-type PTEN gene segments were transfected into glioblastoma cells expressing a mutated form of PTEN. In addition, it was also shown in this study that the PTEN/PI3K/AKT pathway reduced the expression and function of hTERT [17]. A recent study also demonstrated that PTEN suppressed the phosphorylation of various tumor related proteins including hTERT through the PI3K/AKT pathway in renal cell carcinoma [18].

Our previous study has also found that the proliferative capacity of lung adenocarcinoma cells was significantly reduced when the exogenous wild-type PTEN gene was introduced into A549/CDDP cells, which are resistant to cisplatin. Simultaneously, G1 phase arrest was observed and the A549/CDDP cells displayed a considerable improvement in sensitivity to cisplatin [19]. In light of the above, it is reasonable for us to presume that the mechanism by which PTEN inhibits cell proliferation, promotes cell apoptosis, and induces cell cycle arrest in lung adenocarcinoma A549 cells may be related to the downregulation of $h T E R T$ expression and that the PI3K/AKT pathway might be implicated in this process.

\section{Materials and Methods}

2.1. Cell Line and Cell Culture. The human lung adenocarcinoma cell line (A549) was purchased from the Cell Center of Xiangya Medical College of Central South University (Changsha, Hunan, China). Cells were cultured in highglucose Dulbecco's modified Eagle's medium (DMEM, Gibco, CA, USA) supplemented with $10 \%$ fetal bovine serum (Gibco, CA, USA), $100 \mathrm{U} / \mathrm{mL}$ penicillin, $100 \mu \mathrm{g} / \mathrm{mL}$ streptomycin sulfate, and $2 \mathrm{mM}$ glutamate in humidified atmosphere of $5 \%$ $\mathrm{CO}_{2}$ at $37^{\circ} \mathrm{C}$.

2.2. Gene Transfection Technology. The null plasmid vector (pGFP), wild-type PTEN plasmid (pGFP-PTEN), and mutant-type PTEN plasmid (pGFP-PTEN-C124A) were generously supplied by Professor Kenneth M. Yamada, American National Institutes of Health. Plasmids were amplified by transformation in E. coli, extracted, and purified using a plasmid DNA extract kit (Macherey-Nagel, Germany). Insert sizes were confirmed by agarose gel electrophoresis. PTEN small interfering RNA (PTEN-siRNA) expressing green fluorescence protein (GFP) were synthesized by Cell Signaling Technology Co. Ltd. (Danvers, MA, USA). Each plasmid or siRNA was mixed with Lipofectamine 2000 reagent
(Invitrogen, Carlsbad, CA, USA) following the manufacturer's instructions and was added to cells at 50\%-70\% confluence. After $4 \mathrm{~h}$ of incubation, the transfection medium was discarded, cells were washed three times with phosphate buffered saline (PBS), and the media were then replaced with serum-containing medium. After $48 \mathrm{~h}$, the transfection efficiency and PTEN gene expression were estimated by fluorescence microscopy and Western blot analysis, respectively.

2.3. MTT. A549 cells were trypsinized and seeded into 96well plates at a density of approximately 4000 cells per well. Twenty-four hours later, adherent cells were transfected with pGFP, pGFP-PTEN, pGFP-PTEN-C124A, and PTENsiRNA and/or exposed to PI3K/AKT inhibitor (LY294002 $10 \mu \mathrm{M}$, Sigma-Aldrich, MO, USA), nontransfected A549 cells served as the control group. During the following one to six days, MTT reagent (3-[4,5-dimethylthiazol-2-yl]2,5-diphenyltetrazolium bromide, Sigma-Aldrich, USA) was added to the cells $(20 \mu \mathrm{L}(5 \mathrm{mg} / \mathrm{mL})$ per well), and cells were then incubated for $4 \mathrm{~h}$ at $37^{\circ} \mathrm{C}$. The cells media were removed and $150 \mu \mathrm{L}$ of DMSO was added to each well followed by gentle shaking of the plates to dissolve the formazan crystals. The optical density (OD) was then measured using a microplate reader at $490 \mathrm{~nm}$.

2.4. Flow Cytometry Assay. The A549 cell apoptosis rate (expressed as a percentage) and cell cycle stage were measured using a PI (Propidium Iodide) staining kit (SigmaAldrich, MO, USA). Cells were collected and fixed in $70 \%$ ice cold ethanol overnight, stained with a mixture of PI and RNase in a $37^{\circ} \mathrm{C}$ water bath shielded from light for $30 \mathrm{~min}$. Cells were then examined by fluorescence microscopy and the data were analyzed using WinMDI v 2.8 software.

2.5. Western Blot. A549 cells were trypsinized and washed three times with PBS before being lysed on ice for $30 \mathrm{~min}$ with RIPA lysis buffer containing protease inhibitors (SigmaAldrich, MO, USA). The lysate was centrifuged at $12,000 \times \mathrm{g}$ at $4^{\circ} \mathrm{C}$ for $20 \mathrm{~min}$. Protein concentrations were measured by the Bradford method. Total protein $(50 \mu \mathrm{g})$ for each sample was separated using $10 \%$ sodium dodecyl sulfatepolyacrylamide gel electrophoresis (SDS-PAGE) and transferred into polyvinylidene difluoride (PVDF) membranes (Merck Millipore Inc., MA, USA) and blocked for $1 \mathrm{~h}$ in TBST containing 5\% skimmed milk. Proteins of interests were detected by incubation with the appropriate antibodies at $4^{\circ} \mathrm{C}$ overnight. The primary antibodies used were as follows: mouse anti-human PTEN (1:100 dilution), rabbit anti-human p-AKT (1:500 dilution), and rabbit anti-human hTERT (1:500 dilution) (Abcam Inc., Cambridge, MA, USA). Secondary antibodies (Santa Cruz Biotechnology, CA, USA) were incubated for $1 \mathrm{~h}$ at room temperature. Proteins were detected by ECL chemiluminescence. Image Lab 4.1 software was used to analyze the protein bands and relative protein expression level was normalized with reference to $\beta$-actin (Abcam Inc., Cambridge, MA, USA).

2.6. Real Time PCR. Total RNA was isolated from cells using Trizol reagent (Invitrogen, Carlsbad, CA, USA) according to 
the manufacturer's protocol. Total RNA ( $1 \mu \mathrm{g})$ was reverse transcribed using a Reverse Transcription kit according to the manufacturers protocol (Promega, WI, USA) (reaction at $42^{\circ} \mathrm{C}$ for $40 \mathrm{~min}$ and denaturation at $99^{\circ} \mathrm{C}$ for $5 \mathrm{~min}$ ). cDNA $(2 \mu \mathrm{L})$ was then amplified using the following conditions: PTEN: 30 cycles of denaturation for $50 \mathrm{~s}$ at $94^{\circ} \mathrm{C}$; annealing for $45 \mathrm{~s}$ at $60^{\circ} \mathrm{C}$; and extension for $7 \mathrm{~min}$ at $72^{\circ} \mathrm{C}$ and $h T E R T$ : 30 cycles of denaturation for $30 \mathrm{~s}$ at $94^{\circ} \mathrm{C}$; annealing for $30 \mathrm{~s}$ at $60^{\circ} \mathrm{C}$; and extension for $45 \mathrm{~s}$ at $72^{\circ} \mathrm{C}$. The PCR product for each sample was separated by $1.5 \%$ agarose gel electrophoresis at $80 \mathrm{~V}$. The $\beta$-actin or GAPDH mRNA expression level was employed as an internal control. The sequence of the primer pairs used is listed below:

PTEN: 5 '-GTAAGGACCAGAGACAAAAAG-3' and $3^{\prime}$-CTTTTTTAGCATCTTGTTCTG-5'

hTERT: $5^{\prime}$-TTTCTGGAGCTGCTTGGGAA-3' and $3^{\prime}$-GAAGAGCCTGAGCAGCTCGA-5'

$\beta$-Actin: $5^{\prime}$-TGAAGTGTGACGTGGACATC-3' and $3^{\prime}$-GGAGGAGCAATGATCTTGAT-5'

GAPDH: $\quad 5^{\prime}$-ACCACAGTCCATGCCATCAC-3' and $3^{\prime}$-TCCACCACCCTGTTGCTGTA-5'

2.7. Statistical Analysis. GraphPad Prism 5 software was used for statistical analysis. All data were obtained from at least three independent experiments. Data in each group is presented as the mean \pm standard deviation $(\chi \pm \mathrm{SD})$, and the difference between groups was analyzed by analysis of variance (ANOVA) or a two-tailed Student's $t$-test. $p$ value less than 0.05 was counted as being statistically different.

\section{Results}

3.1. Effects of Different PTEN Phenotypes on A549 Cell Proliferation, Apoptosis, and Cell Cycle Progression. Our previous report demonstrated that PTEN could regulate cell proliferation, cell cycle, and drug sensitivity to cisplatin in A549/CDDP cells. It has been documented that A549 cells express wild-type PTEN [20, 21]. To further investigate whether different PTEN phenotypes have the same antitumor effect on A549 cells, vector alone (null), wild-type PTEN, a phosphatase-dead PTEN mutant (PTEN-C124A), and PTEN-siRNA were transfected separately into A549 cells using untransfected A549 cells as the control group. Cell proliferation was assessed using an MTT assay whereas cell apoptosis and cell cycle progression were assessed using a flow cytometry assay. As shown in Figure 1(a), the cell growth curves showed logarithmic growth from the first day to the fourth day; after this time, cell proliferation began to plateau. Over the first four days of growth, it was readily apparent that cell proliferation was suppressed in cells overexpressing wild-type PTEN compared to control cells. In contrast, cell proliferation in the PTEN-siRNA cells was significantly enhanced $(p<0.05)$. Furthermore, there was no apparent change in cell proliferation in both the null cells and the phosphatase-dead PTEN cells compared with the control cells $(p>0.05)$. The PI3K/AKT pathway can be blocked by using the AKT inhibitor LY294002. Our results showed that, in control cells, LY294002 treatment also suppressed cell proliferation. In contrast, the enhancement of proliferation seen in the PTEN-siRNA treated cells was blocked by LY294002 treatment.

The flow cytometry data demonstrated that the rate of cell apoptosis was significantly enhanced in cells overexpressing wild-type PTEN, compared with the control cells (15.6 \pm $1.14 \%$ versus $\left.2.27 \pm 0.21 \%,{ }^{* *} p<0.05\right)$. LY294002 of control cells also increased the level of apoptosis to levels similar to those seen in wild-type PTEN overexpressing cells $(10.36 \pm$ $2.7 \%$ versus $\left.2.27 \pm 0.21 \%,{ }^{\triangle} p<0.05\right)$. Similarly, compared with the control cells, there was an increased proportion of G0-G1 phase cells in the wild-type PTEN group $(71.5 \pm 3.22 \%$ versus $\left.47.5 \pm 1.03 \%,{ }^{* *} p<0.05\right)$ and LY294002 treatment of control cells also similarly increased the proportion of cells in the G0-G1 phase $(63.1 \pm 2.64 \%$ versus $47.5 \pm 1.03 \%$, $\left.{ }^{\triangle} p<0.05\right)$. On the contrary, both the rate of cell apoptosis $\left(0.55 \pm 0.15 \%\right.$ versus $\left.2.27 \pm 0.21 \%,{ }^{*} p<0.05\right)$ and the proportion of cells in the G0-G1 phase $(30.5 \pm 1.89 \%$ versus $\left.47.5 \pm 1.03 \%,{ }^{\#} p<0.05\right)$ were significantly reduced in the PTEN-siRNA group compared to the control group. Treatment of PTEN-siRNA cells with LY294002 attenuated both the decrease in rate of cell apoptosis $(6.71 \pm 2.47$ versus $\left.0.55 \pm 0.15 \%,{ }^{\circ} p<0.05\right)$ and the decrease in percent of cells in the G0-G1 phase $(59.3 \pm 2.77 \%$ versus $30.5 \pm 1.89 \%$, $\left.{ }^{\circ} p<0.05\right)$ caused by the PTEN-siRNA. Moreover, compared with control group, there was no statistical difference in the rate of cell apoptosis for the null cells $(2.13 \pm 0.36 \%$ versus $\left.2.27 \pm 0.21 \%,{ }^{*} p>0.05\right)$ or the phosphatase-dead PTEN cells $\left(2.31 \pm 0.57 \%\right.$ versus $\left.2.27 \pm 0.21 \%,{ }^{\square} p>0.05\right)$. Similar observations were made for the percentage of cells in the G0-G1 phase $(45.6 \pm 2.22 \%$ (null) versus $47.5 \pm 1.03 \%$ (control), ${ }^{*} p>0.05$, and $50.3 \pm 3.27 \%$ (phosphatase-dead PTEN) versus $47.5 \pm 1.03 \%$ (control), ${ }^{\square} p>0.05$ ) (Figures $1(\mathrm{~b})$ and $1(\mathrm{c})$ ). These results suggested that wild-type PTEN, but not a phosphatase-dead PTEN mutant, was capable of suppressing cell proliferation, inducing cell apoptosis, and arresting the cell cycle in adenocarcinoma A549 cells and that the PI3K/AKT pathway possibly participated in these effects.

3.2. PTEN Negatively Regulates hTERT by Inhibiting the PI3K/AKT Pathway. As shown in Figure 1, the role of PTEN as a tumor suppressor in A549 cells has been convincingly demonstrated by its effects on cell proliferation, apoptosis, and cell cycle arrest. In light of previous reports showing that PTEN can negatively regulate hTERT in hepatocellular carcinoma, renal carcinoma, and glioma cells and that PI3K/AKT acts downstream of PTEN, we hypothesized that PTEN might inhibit tumor progression by suppressing the $\mathrm{PI} 3 \mathrm{~K} / \mathrm{AKT} / \mathrm{hTERT}$ pathway.

In order to investigate this hypothesis, we examined the mRNA and protein expression level of PTEN, hTERT, and p-AKT in cells expressing different levels of PTEN, or a phosphatase-dead PTEN mutant, along with the combination of treatment with PI3K/AKT pathway inhibitor LY294002. As shown in Figures 2(a) and 2(c), there was no detectable difference in the mRNA and protein expression level of PTEN between the control group and the null plasmid transfected 


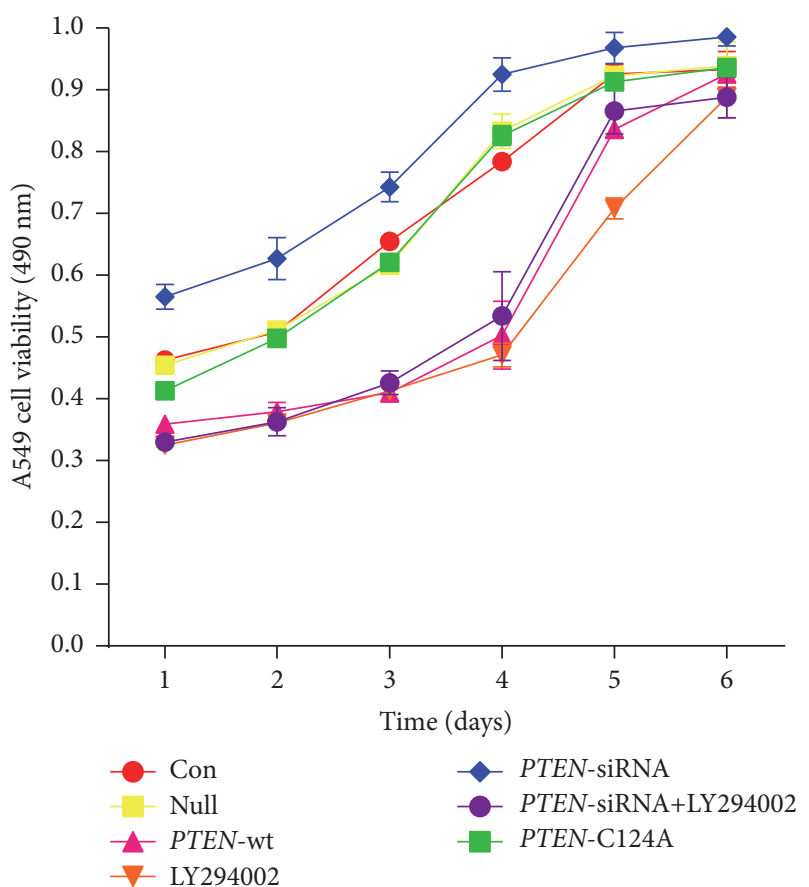

(a)

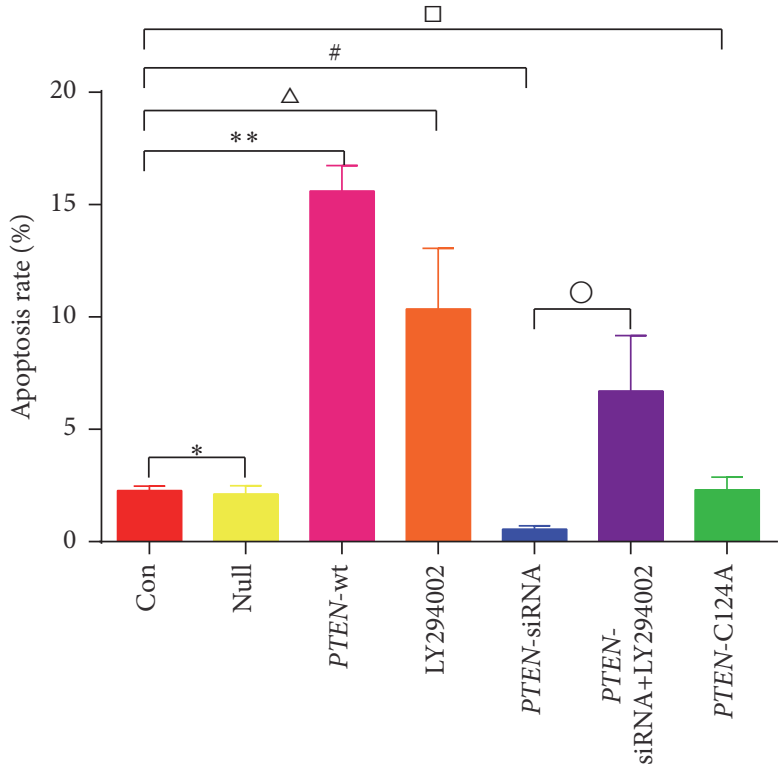

(b)

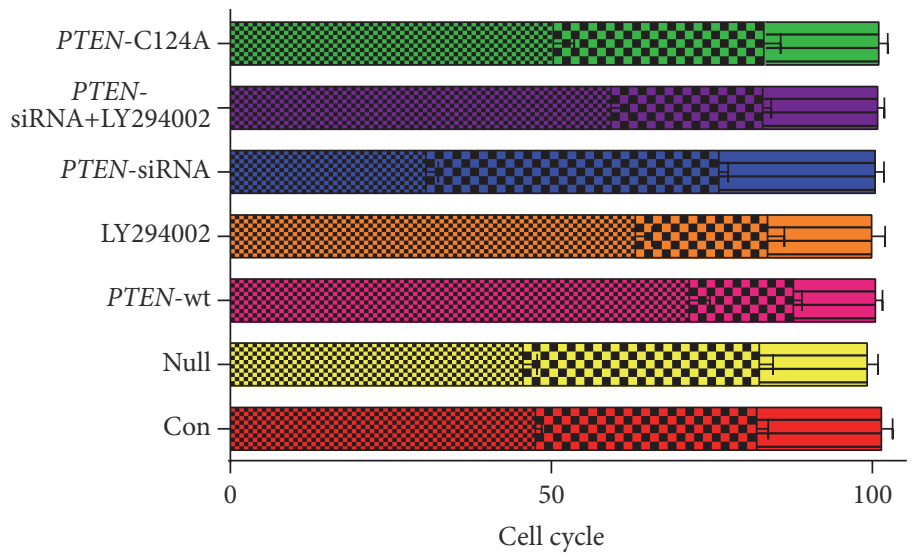

伖 G0-G1

W S

$\exists \mathrm{G} 2-\mathrm{M}$

(c)

FIGURE 1: Effects of different PTEN phenotypes on A549 cell proliferation, apoptosis, and cell cycle progression. A549 cells were transfected with pGFP (null), pGFP-PTEN (PTEN-wt), pGFP-phosphatase-dead PTEN (PTEN-C142A), or PTEN-siRNA in the presence or absence of LY294002 $(10 \mu \mathrm{M})(P T E N$-siRNA and PTEN-siRNA+LY294002). Untransfected A549 cells were used as the control group (con) and these cells were also treated with LY294002 (10 $\mathrm{MM})(\mathrm{LY} 294002)$. Cell viability (a), the cellular apoptosis rate (b), and cell cycle progression (c) were measured by MTT and flow cytometry, respectively $\left({ }^{*} p>0.05,{ }^{* *} p<0.05,{ }^{\Delta} p<0.05,{ }^{\#} p<0.05,{ }^{\square} p>0.05\right.$, and $\left.{ }^{\circ} p<0.05\right)$.

cells, which demonstrated that the plasmid vector did not affect PTEN expression. As expected, upregulation of PTEN at both the mRNA and protein levels could be observed in cells transfected with wild-type PTEN as well as in cells transfected with the phosphatase-dead mutant PTEN. In cells transfected with a PTEN-siRNA, also as expected, lower levels of PTEN could be detected at both the mRNA and protein levels. Treatment of cells with LY294002 did not affect PTEN expression, which we believe is evidence that PI3K/AKT is located downstream of PTEN.
In this study, we also found that the mRNA and protein levels of $h T E R T$ were considerably lower in A549 cells transfected with wild-type PTEN than in the control group $(p<0.05)$. Conversely, dramatically elevated $h T E R T$ mRNA and protein level could be observed in A549 cells transfected with a PTEN-siRNA compared to the control group $(p<$ $0.05)$. These results confirm that PTEN also negatively regulates $h T E R T$ in lung adenocarcinoma cells. Although the PTEN mRNA and protein levels increased after A549 cells were transfected with the phosphatase-dead PTEN mutant 


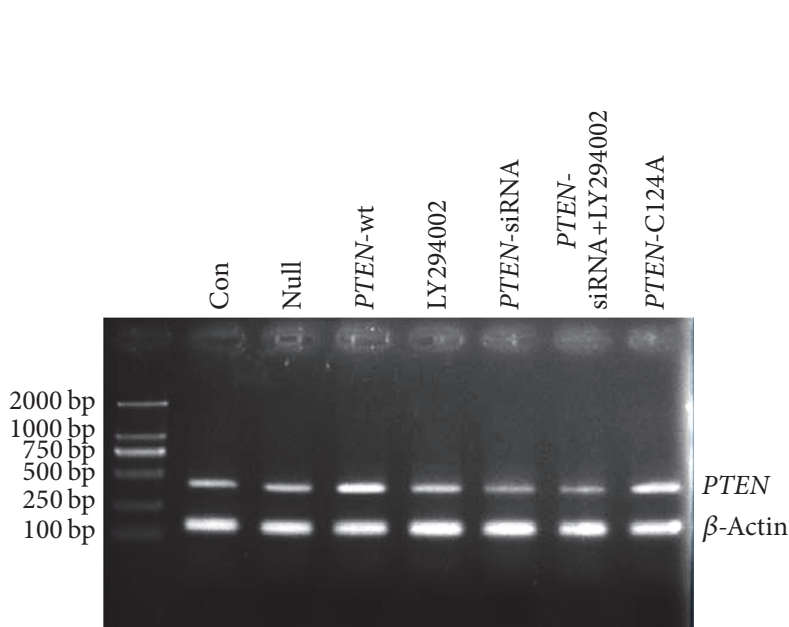

(a)

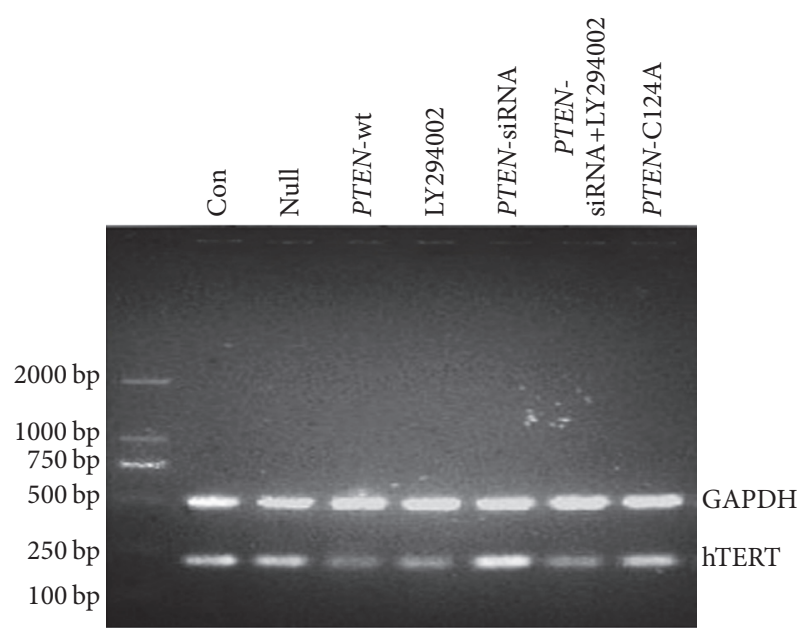

(b)

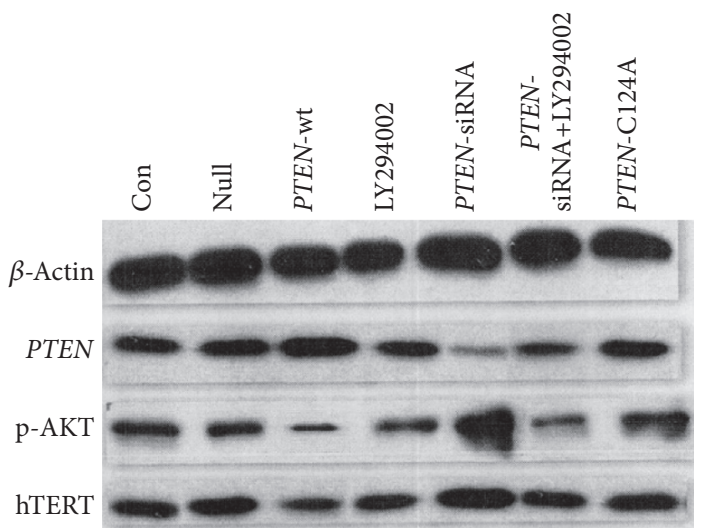

(c)

FIGURE 2: PTEN negatively regulates $h T E R T$ by inhibiting the PI3K/AKT pathway. Untransfected A549 cells (con) or A549 cells transfected with either pGFP (null), pGFP-PTEN (wild-type PTEN), pGFP-PTEN-C124A (phosphatase-dead PTEN), or PTEN-siRNA and/or exposed to the AKT inhibitor (LY294002 $10 \mu \mathrm{M}$ ) were examined for the expression of PTEN (a) and $h T E R T$ (b) mRNA by RT-PCR, as well as for the protein levels of PTEN, hTERT, and p-AKT (c) by Western blot.

(PTEN-C124A), this was not accompanied by a change in hTERT expression levels $(p>0.05)$, confirming that the phosphatase-dead PTEN is nonfunctional and that only wildtype PTEN plays a role in suppressing $h T E R T$ expression (see Figures 2(b) and 2(c)).

Intriguingly, we also found that, compared to control cells, the phosphorylation levels of AKT were decreased in cells overexpressing wild-type PTEN, while they were increased in cells transfected with a PTEN-siRNA. These data highlight the fact that PI3K/AKT is inhibited by wild-type PTEN but not by a phosphatase-dead mutant PTEN in these lung adenocarcinoma cells. Furthermore, we demonstrated that the PI3K/AKT pathway blocker LY294002 could mimic the effects of wild-type PTEN overexpression. Both the mRNA and the protein level of $h T E R T$ were remarkably lower in A549 cells after exposure to LY294002 alone. Treatment of cells expressing a PTEN directed siRNA with LY294002 abrogated the upregulation of hTERT expression caused by the PTEN-siRNA $(p<0.05)$ (see Figures 2(b) and 2(c)). Collectively, these data, as well as previously published results, reveal that the PTEN gene act as a tumor suppressor by inhibiting cell proliferation, promoting cell apoptosis, and inducing cell cycle arrest in human lung adenocarcinoma in vitro and that these effects were somewhat mediated through a negative regulation of the PI3K/AKT/hTERT pathway.

\section{Discussion}

Although it is well known that the PTEN gene plays a pivotal role in suppressing tumor development by negatively regulating the hTERT gene in multiple tumor cells, it remains to be discovered whether similar effects of PTEN on the hTERT gene also occur in lung cancer. In this study, we focused on the human lung cancer A549 cell line and evaluated the function of PTEN gene on cell proliferation, apoptosis, and cell cycle arrest by over- or underexpressing wild-type PTEN and comparing effects to those seen with phosphatase-dead mutant PTEN.

Data from previous studies in glioma, endometrial cancer, and other tumors suggested that the exogenous wildtype PTEN gene can profoundly inhibit the growth of tumor cells, promote cellular apoptosis, and cause cell cycle arrest 
at the G1 phase [22-27]. Our study, in lung cancer cells, now also confirms these findings. Although the A549 cell line expresses low levels of the PTEN gene [28, 29], these levels can be further increased by transfection with the exogenous wild-type PTEN and this leads to suppression of cell proliferation. Bruni and his colleagues [30] found that the expression of exogenous wild-type PTEN can inhibit tumor growth independent of whether the cells express the endogenous PTEN gene or not and that the inhibitory effect is more obvious when the endogenous PTEN gene is completely deleted. On the contrary, cell proliferation and apoptosis are unchanged in A549 cells expressing a phosphatase-dead mutant PTEN gene (PTEN-C124A) $[5,31,32]$. These data highlight the fact that the phosphatase activity of the PTEN gene is indispensable for the effects of PTEN on restraining cancer cell growth as well as promoting apoptosis.

Here, we further analyzed the relationship between the PTEN and hTERT genes in A549 cells. The results showed that the mRNA and protein levels of PTEN increased after transfection of lung adenocarcinoma A549 cells with the wild-type PTEN plasmid and that at the same time hTERT mRNA and protein expression levels were reduced. However, there were no obvious changes of the hTERT mRNA and protein expression observed in A549 cells transfected the mutant-type PTEN plasmid. In addition, we found that the $h T E R T$ mRNA and protein expression levels increased when the PTEN gene was silenced using a PTEN directed siRNA. These data suggest that the expression level of $h T E R T$ is inversely associated with the activity of the wild-type PTEN gene.

The $h T E R T$ gene is considered to be the key rate limiting factor, which regulates the activity of telomerase, and its expression level may indirectly reflect the activity of telomerase. It plays a critical role in the process of development of tumor by inducing the clonal growth of cell by bypassing the process of replicative senescence thereby contributing to malignant immortalization $[2,14,18]$. An earlier study has reported that, in approximately $85 \%$ of people with cancer, telomerase activity could be detected in tumor tissues, whereas telomerase activity was detected in only about $4 \%$ of normal tissues adjacent to the tumor or in benign lesions [33]. Increased telomerase activity can suppress tumor cell apoptosis by affecting DNA stability and through signal transduction pathways [34]. Concordantly, it has also been demonstrated that the reduction of hTERT expression using an $h T E R T$ siRNA inhibited telomerase activity and accelerated cell apoptosis in lung cancer [35], further strengthening our hypothesis that $P T E N$ suppresses the activity of telomerase by decreasing the expression of $h T E R T$, leading to the inhibition of cell proliferation and the promotion cell apoptosis in lung adenocarcinoma A549 cells.

The PTEN gene participates in a myriad of physiological and pathological processes and its biological effects mainly depend on its interaction with downstream signaling molecules. In view of the evidence that the PTEN-PI3K/AKT$h T E R T$ axis is a commonly operative pathway in various carcinoma models, we presumed that the PI3K/AKT pathway might be implicated in the regulation of $h T E R T$ by PTEN in lung cancer. Our results show that the levels of phosphorylated AKT (p-AKT) were reduced and that cell proliferation was inhibited in A549 cells overexpressing wildtype PTEN compared to control cells. The opposite effects were observed in cells transfected with a PTEN directed siRNA. In order to clarify if AKT plays a central role in this process, we used LY294002 a PI3K/AKT pathway inhibitor that inhibits the AKT pathway [36]. As expected, following LY294002 treatment, the AKT protein phosphorylation levels decreased. Importantly, LY294002 treatment of A549 cells decreased cellular proliferation, increased the apoptosis rate, and increased the percentage of cells arrested at G1. These effects were very similar in magnitude to those observed by overexpression of PTEN itself. LY294002 treatment of cells expressing a PTEN directed siRNA also profoundly suppressed the enhanced cell proliferation, reduced cellular apoptosis, and decreased cell cycle arrest seen in the PTENsiRNA transfected cells alone. Based on these data, we have verified that the PI3K/AKT pathway indeed plays a central role in the mediating of the effects of PTEN on cell proliferation, apoptosis, and cell cycle arrest in A549 cells.

As an essential signal transduction protein, AKT plays a central role in the PI3K/AKT cell survival signaling pathway in cancer cells, and it also plays a crucial role in the cell survival mechanisms and signal transduction pathways mediating tumorigenesis. Only the phosphorylated form of AKT (namely, p-AKT) has biological activity [37]. This can be attributed to two factors. First, phosphorylated AKT prevents cell apoptosis by several mechanisms, including direct phosphorylation, the preapoptosis protein $\mathrm{BAD}$, direct phosphorylation of the forkhead transcription factor FKHR-L1, and reducing the protease activity of caspase-9. AKT activation depends on the production of PIP3 by PI3K, and PTEN dephosphorylates PIP3 at the $3^{\prime}$ position, thereby preventing phosphorylation and activation of AKT and subsequently leading to the activation of downstream apoptosis signaling pathways and an increase in apoptosis [38-41]. Second, PTEN is also able to downregulate the cyclin-dependent kinase (CDK) by virtue of its protein phosphatase activity. In addition, PTEN can block the phosphorylation of CDKIs (cyclin-dependent kinase inhibitors) by AKT, allowing the entry of CDKIs into the nucleus and thereby suppressing the function of CDKs and impeding cell cycle progression [34, 42-44].

In addition, data from our study showed that hTERT mRNA and protein expression were clearly reduced when we utilized LY294002 to block the PI3K/AKT pathway in A549 cells. This same reduction was also seen in A549 cells overexpressing wild-type PTEN. Treatment of A549 cells expressing a PTEN directed siRNA with LY294002 abrogated the upregulation of hTERT expression seen in untreated cells. These data, along with the data on cell proliferation, apoptosis, and cell cycle arrest, show that inhibition of the PI3K/AKT pathway by LY294002 can mimic the anticancer effect of wild-type PTEN. In agreement with the present study, another study using the cervical cancer HeLa cell line treated with LY294002 showed that expression of hTERT can also be affected by inhibition of the PI3K/AKT pathway. Another study in endometrial carcinoma also showed that PTEN could reduce hTERT mRNA expression by the 
PI3K/AKT pathway $[45,46]$. Past studies have pointed out that hTERT, as a telomerase complex catalytic unit, can be activated in a variety of ways, including $\mathrm{PKB} / \mathrm{AKT}$ phosphorylation [10]. Although the mechanism by which the PI3K/AKT pathway upregulates hTERT is unclear, it has been suggested that it could be mediated by direct phosphorylation of serine residues in hTERT by p-AKT [13].

\section{Conclusions}

In light of the above findings, we conclude that downregulation of the PI3K/AKT/hTERT pathway may be one of the mechanisms by which the PTEN gene can act as a tumor suppressor in lung adenocarcinoma A549 cells. Further studies in animal model will still be needed to pave the way for future lung cancer treatment and prevention strategies.

\section{Competing Interests}

The authors declare that there are no competing interests regarding the publication of this paper.

\section{Acknowledgments}

This work was supported by funding from the National Natural Science Foundation of China (81572284) and the Key Program of Hunan province Science and Technology Hall (2015SK20662).

\section{References}

[1] X.-B. Li, Y. Yang, H.-Q. Zhang et al., "High levels of phosphatase and tensin homolog expression predict favorable prognosis in patients with non-small cell lung cancer," European Review for Medical and Pharmacological Sciences, vol. 19, no. 12, pp. 22312239, 2015.

[2] E. J. Kim, S. Y. Lee, T. R. Kim et al., "TSPYL5 is involved in cell growth and the resistance to radiation in A549 cells via the regulation of $\mathrm{p} 21 \mathrm{WAF} 1 / \mathrm{Cip} 1$ and PTEN/AKT pathway," Biochemical and Biophysical Research Communications, vol. 392, no. 3, pp. 448-453, 2010.

[3] L. Wang, L.-E. Wang, L. Mao, M. R. Spitz, and Q. Wei, "A functional variant of tandem repeats in human telomerase gene was associated with survival of patients with early stages of nonsmall cell lung cancer," Clinical Cancer Research, vol. 16, no. 14, pp. 3779-3785, 2010.

[4] M. Stražišar, V. Mlakar, and D. Glavač, "The expression of COX2, hTERT, MDM2, LATS2 and S100A2 in different types of nonsmall cell lung cancer (NSCLC)," Cellular and Molecular Biology Letters, vol. 14, no. 3, pp. 442-456, 2009.

[5] J. Li, C. Yen, D. Liaw et al., "PTEN, a putative protein tyrosine phosphatase gene mutated in human brain, breast, and prostate cancer," Science, vol. 275, no. 5308, pp. 1943-1947, 1997.

[6] G. L. Mutter, "Commentary-PTEN, a protean tumor suppressor," The American Journal of Pathology, vol. 158, no. 6, pp. 18951898, 2001.

[7] Z.-Y. Cheng, X.-L. Guo, S.-H. Li et al., “The role of PTEN-FAK signaling pathway in metastasis and invasive ability of leukemia cells," Zhonghua Xue Ye Xue Za Zhi, vol. 30, no. 2, pp. 115-120, 2009.
[8] S. Bouali, A.-S. Chrétien, C. Ramacci, M. Rouyer, P. Becuwe, and J.-L. Merlin, "PTEN expression controls cellular response to cetuximab by mediating PI3K/AKT and RAS/RAF/MAPK downstream signaling in KRAS wild-type, hormone refractory prostate cancer cells," Oncology Reports, vol. 21, no. 3, pp. 731$735,2009$.

[9] Q.-B. She, D. B. Solit, Q. Ye, K. E. O’Reilly, J. Lobo, and $\mathrm{N}$. Rosen, "The BAD protein integrates survival signaling by EGFR/MAPK and PI3K/Akt kinase pathways in PTEN-deficient tumor cells," Cancer Cell, vol. 8, no. 4, pp. 287-297, 2005.

[10] Q. Gao, F. Ye, X. Xia et al., "Correlation between PTEN expression and PI3K/Akt signal pathway in endometrial carcinoma," Journal of Huazhong University of Science and TechnologyMedical Science, vol. 29, no. 1, pp. 59-63, 2009.

[11] J. Ding, B. Ningand, W. Gong et al., "Cyclin D1 induction by benzo[a]pyrene-7,8-diol-9,10-epoxide via the phosphatidylinositol 3-kinase/Akt/MAPK- and $\mathrm{p} 70^{s 6 k}$-dependent pathway promotes cell transformation and tumorigenesis," The Journal of Biological Chemistry, vol. 284, no. 48, pp. 33311-33319, 2009.

[12] M. Tamura, J. Gu, K. Matsumoto, S.-I. Aota, R. Parsons, and K. M. Yamada, "Inhibition of cell migration, spreading, and focal adhesions by tumor suppressor PTEN," Science, vol. 280, no. 5369, pp. 1614-1617, 1998.

[13] A. Kimura, M. Ohmichi, J. Kawagoe et al., "Induction of hTERT expression and phosphorylation by estrogen via Akt cascade in human ovarian cancer cell lines," Oncogene, vol. 23, no. 26, pp. 4505-4515, 2004.

[14] W. Li, Y. Ni, Z. Tu, S. Wu, Z. Wu, and S. Zheng, "Study of telomerase activity in pleural lavage fluid specimens in patients with non-small-cell lung cancer and its clinical significance," European Journal of Cardio-Thoracic Surgery, vol. 36, no. 3, pp. 460-464, 2009.

[15] D. C. F. Sealey, L. Zheng, M. A. S. Taboski, Jennifer Cruickshank, M. Ikura, and L. A. Harrington, "The N-terminus of hTERT contains a DNA-binding domain and is required for telomerase activity and cellular immortalization," Nucleic Acids Research, vol. 38, no. 6, pp. 2019-2035, 2010.

[16] X. Zhou, H. Zhu, and J. Lu, "PTEN and hTERT gene expression and the correlation with human hepatocellular carcinoma," Pathology Research and Practice, vol. 211, no. 4, pp. 316-319, 2015.

[17] X.-X. Tian, J. C. S. Pang, S. S. T. To, and H.-K. Ng, "Restoration of wild-type PTEN expression leads to apoptosis, induces differentiation, and reduces telomerase activity in human glioma cells," Journal of Neuropathology and Experimental Neurology, vol. 58, no. 5, pp. 472-479, 1999.

[18] R. T. Sitaram, C. J. Cairney, P. Grabowski et al., "The PTEN regulator DJ-1 is associated with hTERT expression in clear cell renal cell carcinoma," International Journal of Cancer, vol. 125, no. 4, pp. 783-790, 2009.

[19] L.-F. Min, L.-L. He, C. Qiong, Y. Qiao, and M.-X. Xie, "Magnetic iron oxide nanoparticles carrying PTEN gene to reverse cisplatin-resistance of A549/CDDP cell lines," Journal of Central South University of Technology, vol. 19, no. 2, pp. 331-339, 2012.

[20] J. Chen, H. Bi, J. Hou et al., "Atorvastatin overcomes gefitinib resistance in KRAS mutant human non-small cell lung carcinoma cells," Cell Death and Disease, vol. 4, no. 9, article e814, 2013.

[21] Y.-K. Yoon, H.-P. Kim, S.-W. Han et al., "KRAS mutant lung cancer cells are differentially responsive to MEK inhibitor due to AKT or STAT3 activation: implication for combinatorial approach," Molecular Carcinogenesis, vol. 49, no. 4, pp. 353-362, 2010. 
[22] F. B. Furnari, H. Lin, H.-J. S. Huang, and W. K. Cavenee, "Growth suppression of glioma cells by PTEN requires a functional phosphatase catalytic domain," Proceedings of the National Academy of Sciences of the United States of America, vol. 94, no. 23, pp. 12479-12484, 1997.

[23] M. A. Davies, D. Koul, H. Dhesi et al., "Regulation of Akt/PKB activity, cellular growth, and apoptosis in prostate carcinoma cells by MMAC/PTEN," Cancer Research, vol. 59, no. 11, pp. 2551-2556, 1999.

[24] L.-P. Weng, J. L. Brown, and C. Eng, "PTEN induces apoptosis and cell cycle arrest through phosphoinositol-3-kinase/Aktdependent and -independent pathways," Human Molecular Genetics, vol. 10, no. 3, pp. 237-242, 2001.

[25] A. Di Cristofano and P. P. Pandolfi, “The multiple roles of PTEN in tumor suppression,” Cell, vol. 100, no. 4, pp. 387-390, 2000.

[26] L.-P. Weng, O. Gimm, J. B. Kum et al., "Transient ectopic expression of PTEN in thyroid cancer cell lines induces cell cycle arrest and cell type-dependent cell death," Human Molecular Genetics, vol. 10, no. 3, pp. 251-258, 2001.

[27] L.-P. Weng, W. M. Smith, P. L. M. Dahia et al., "Pten suppresses breast cancer cell growth by phosphatase activity- dependent arrest followed by cell death," Cancer Research, vol. 59, no. 22, pp. 5808-5814, 1999.

[28] L. Song, D. Li, Y. Gu et al., "MicroRNA-126 targeting PIK3R2 inhibits NSCLC A549 cell proliferation, migration, and invasion by regulation of PTEN/PI3K/AKT pathway," Clinical Lung Cancer, vol. 17, no. 5, pp. e65-e75, 2016.

[29] C.-L. Chen, T.-H. Chiang, P.-C. Tseng, Y.-C. Wang, and C.-F. Lin, "Loss of PTEN causes SHP2 activation, making lung cancer cells unresponsive to IFN- $\gamma$," Biochemical and Biophysical Research Communications, vol. 466, no. 3, pp. 578-584, 2015.

[30] P. Bruni, A. Boccia, G. Baldassarre et al., "PTEN expression is reduced in a subset of sporadic thyroid carcinomas: evidence that PTEN-growth suppressing activity in thyroid cancer cells is mediated by p27(kipl)," Oncogene, vol. 19, no. 28, pp. 31463155, 2000.

[31] K. M. Yamada and M. Araki, “Tumor suppressor PTEN: modulator of cell signaling, growth, migration and apoptosis," The Journal of Cell Science, vol. 114, no. 13, pp. 2375-2382, 2001.

[32] H. Wu, S. Wang, D. Weng et al., "Reversal of the malignant phenotype of ovarian cancer A2780 cells through transfection with wild-type PTEN gene," Cancer Letters, vol. 271, no. 2, pp. 205-214, 2008.

[33] V. C. H. Wong, J. Ma, and C. E. Hawkins, "Telomerase inhibition induces acute ATM-dependent growth arrest in human astrocytomas," Cancer Letters, vol. 274, no. 1, pp. 151-159, 2009.

[34] Y. Cong and J. W. Shay, "Actions of human telomerase beyond telomeres," Cell Research, vol. 18, no. 7, pp. 725-732, 2008.

[35] X. Dong, A. Liu, C. Zer et al., "siRNA inhibition of telomerase enhances the anti-cancer effect of doxorubicin in breast cancer cells," BMC Cancer, vol. 9, article 133, 2009.

[36] Y.-L. Yang, P.-H. Huang, H.-C. Chiu et al., "Histone deacetylase inhibitor AR42 regulates telomerase activity in human glioma cells via an Akt-dependent mechanism," Biochemical and Biophysical Research Communications, vol. 435, no. 1, pp. 107-112, 2013.

[37] F. Cappuzzo, E. Magrini, G. L. Ceresoli et al., "Akt phosphorylation and gefitinib efficacy in patients with advanced non-smallcell lung cancer," Journal of the National Cancer Institute, vol. 96, no. 15, pp. 1133-1141, 2004.
[38] S. Persad, S. Attwell, V. Gray et al., "Inhibition of integrin-linked kinase (ILK) suppresses activation of protein kinase B/Akt and induces cell cycle arrest and apoptosis of PTEN-mutant prostate cancer cells," Proceedings of the National Academy of Sciences of the United States of America, vol. 97, no. 7, pp. 3207-3212, 2000.

[39] H. Huang, J. C. Cheville, Y. Q. Pan, P. C. Roche, L. J. Schmidt, and D. J. Tindall, "PTEN induces chemosensitivity in PTENmutated prostate cancer cells by suppression of Bcl-2 expression," The Journal of Biological Chemistry, vol. 276, no. 42, pp. 38830-38836, 2001.

[40] L. Li, F. Liu, R. A. Salmonsen et al., "PTEN in neural precursor cells: regulation of migration, apoptosis, and proliferation," Molecular and Cellular Neuroscience, vol. 20, no. 1, pp. 21-29, 2002.

[41] M. H. Cardone, N. Roy, H. R. Stennicke et al., "Regulation of cell death protease caspase- 9 by phosphorylation," Science, vol. 282, no. 5392, pp. 1318-1321, 1998.

[42] H. Wu, V. Goel, and F. G. Haluska, "PTEN signaling pathways in melanoma," Oncogene, vol. 22, no. 20, pp. 3113-3122, 2003.

[43] A. R. Gottschalk, D. Basila, M. Wong et al., "p27Kip1 is required for PTEN-induced G1 growth arrest," Cancer Research, vol. 61, no. 5, pp. 2105-2111, 2001.

[44] H.-J. An, Y.-H. Lee, N.-H. Cho et al., "Alteration of PTEN expression in endometrial carcinoma is associated with downregulation of cyclin-dependent kinase inhibitor, p27," Histopathology, vol. 41, no. 5, pp. 437-445, 2002.

[45] X.-X. Yu, Y.-A. Shi, L.-H. Zhang, Y.-S. Wang, and S. Wu, "Telomerase activity, PI3K/AKT signaling pathway and cellular biological behavior in HeLa cell line," Chinese Journal of Pathology, vol. 37, no. 5, pp. 323-327, 2008.

[46] C. Zhou, V. L. Bae-Jump, Y. E. Whang, P. A. Gehrig, and J. F. Boggess, "The PTEN tumor suppressor inhibits telomerase activity in endometrial cancer cells by decreasing hTERT mRNA levels," Gynecologic Oncology, vol. 101, no. 2, pp. 305-310, 2006. 


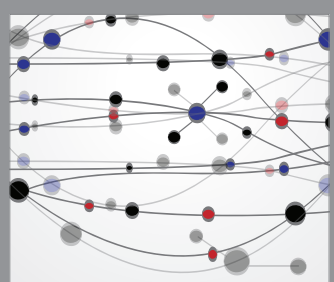

The Scientific World Journal
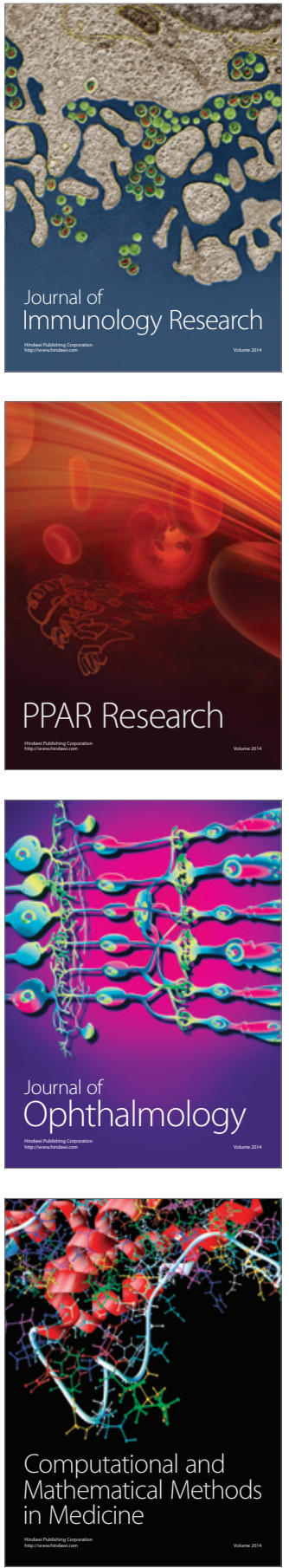

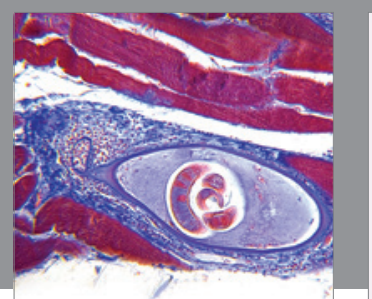

Gastroenterology Research and Practice

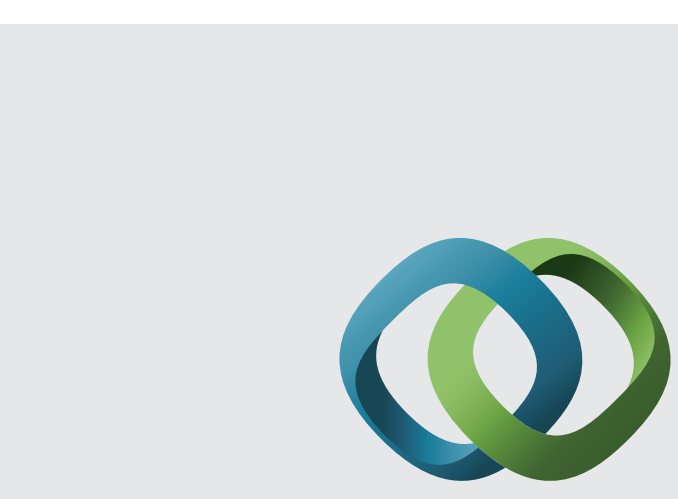

\section{Hindawi}

Submit your manuscripts at

http://www.hindawi.com
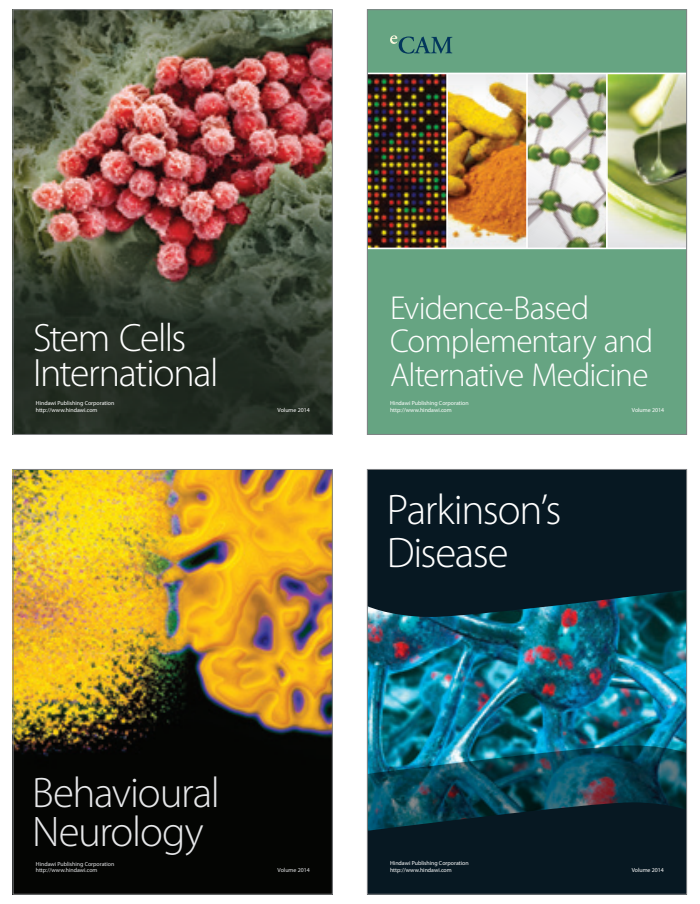
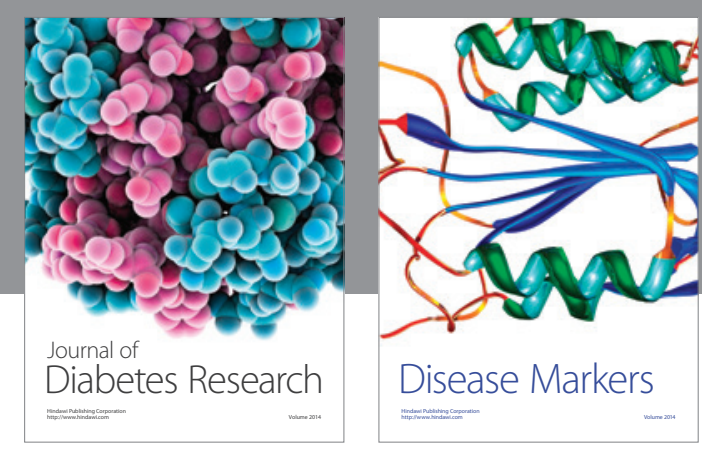

Disease Markers
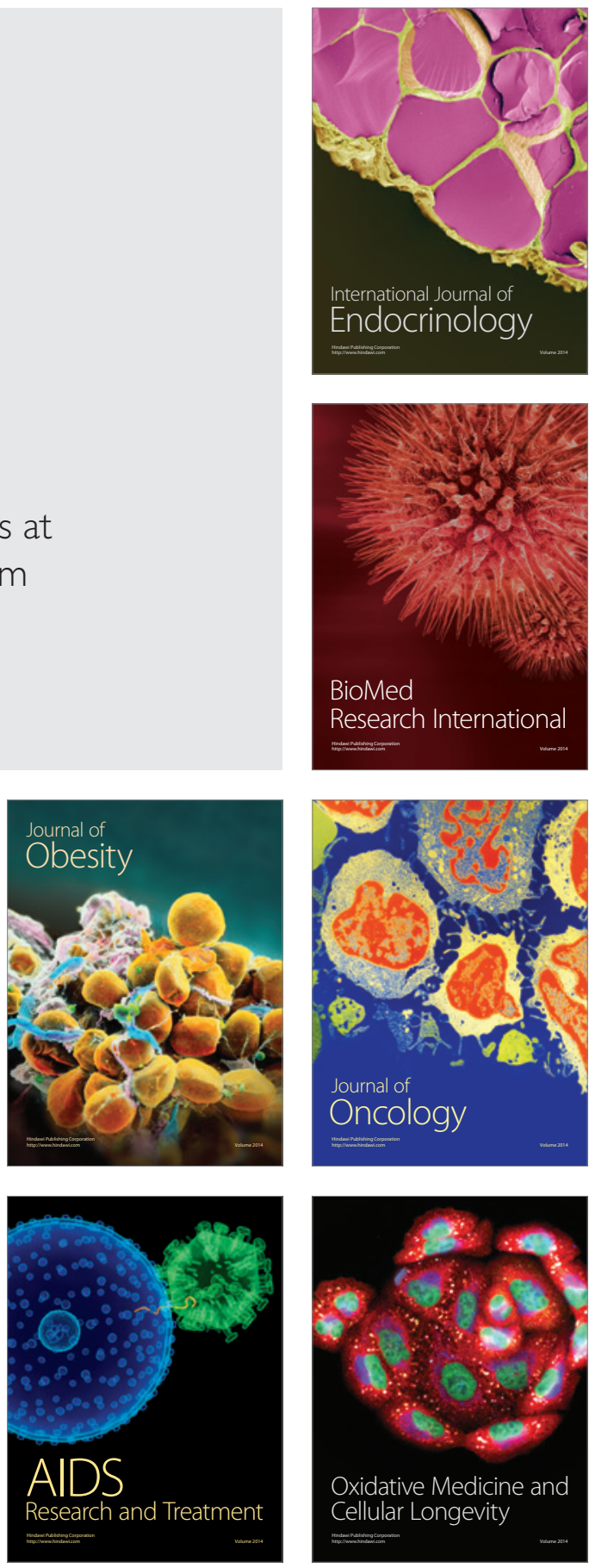